\title{
The Problems and Solutions to the Building of the New-type Rural Cooperative Medical Workforce
}

\author{
Lidan Wang, Yu Zhang, Yahui Zhang, Yayun Yu, Leilei Liu \& Chunyan Mo \\ Management School, Wuhan University of Science and Technology \\ Wuhan 430081, China \\ E-mail:1044686958@qq.com
}

Received: March 20, $2011 \quad$ Accepted: May 6, 2011 doi:10.5539/ass.v7n9p78

\begin{abstract}
In order to improve the rural medical conditions, China has started to try a new policy ---- New-type Rural Cooperative Medical Insurance from July, 2003, which has greatly improved rural medical facilities and alleviated the burden of the rural medical treatment. Rural health care reform has made great achievements. However, research shows that there are still some aspects which are worthy of attention and improvement. Focusing on the collection, collation and research of the survey data, we have found some relatively urgent issues, particularly on the medical team-building, such as an aging medical staff term; what's more, the quality of the medical staff issues should be improved. From the perspective of the doctors, we are analyzing and proposing solutions to these issues. It is significant for the sustainable development of the New-type Rural Cooperative Medical Insurance.
\end{abstract}

Keywords: New-type rural cooperative medical insurance service policy, Medical team, Development

Since piloted in July, 2003, New-type rural cooperative medical service policies have already been implemented for seven years; it has received initial success in the basic level in the country and has been praised widely. On one hand, China is trying to form a pattern of resource allocation that "Attaches importance to the countryside for medical and health work". On the other hand, our country is combining it with building the three levels of public hygiene and medical service network, and establishing the new-type rural cooperative medical service construction, implementing the medical team that is relying on rural communities, such as rural doctor teams. From the current situation, the farmers obviously have got the benefits to see doctors and to get medicine. The whole new-type cooperative medical service system has been well received and welcomed. But during our research, we found a central question which is extant and needed to be solved: the construction and development issues of the rural cooperative medical and health team.

New-type rural cooperative medical service system is a national policy that brings benefits to both people and our country. It needs healthy and stable developments. However, without a solid medical team to support it, it would eventually be lost and lacking of sustainability.

\section{The present situation of the cooperative medical service staff}

\subsection{The formation of the cooperative medical service team}

The rural doctors were born in the 1950s and 1960s, it was so-called "Barefoot Doctor". After the founding of the Peoples Republic of China (PRC), because of the poor conditions of the medical and health factors in the countryside, all types of diseases were widely spread. In serious shortage of medical cases, according to Chairman Mao's "626 indication", that is "Attach importance to the countryside for the medical and health work". Government departments began paying much more attention to the health and medical fields in the countryside and snatched from the basic level. Thus, in order to solve the shortage of medical workers, and improve the poor medical and health services condition, the country and district health bureau set up classes and began to recruit not off-job farmers and the "half doctor and half farmer". Since then, the community began to train peasants to be doctors and not off-job medical workers. After the training, they would take an exam, and the one who passed the exam would take the occupation of "rural doctor", while others would be called rural health workers. As the magazine called "Red Flag" published a report that is entitled as "The direction of the medical education revolution based on the growth of the barefoot doctor", which was reproduced in other newspapers in September 1968, the title, barefoot doctor, was well known to everyone in China. In 1977, 85\% of the production 
brigade had carried out Rural Cooperative Medical Insurance, the number of barefoot doctors were more than 150 million. In the 1980s, the cooperative medical service system was broken off; barefoot doctors became individual doctors and got obligations to look after the people who lived in the countryside. They began working at the basic level in rural areas. With the economic situation continuously developing, constantly improving of health conditions, it gradually formed a structure of one clinic one doctor per village. Combined Chinese medical means with western medical treatment, the level of the standard of the rural medical service had been greatly improved.

\subsection{The contribution of the medical team}

At the beginning, early barefoot doctors wrote an instruction manual for the guidance of health officers. Up to now, barefoot doctors have made great contributions to the new-type rural cooperative medical Insurance. In the 1950s and 1960s, those barefoot doctors took on the healthcare and took charge of anti-epidemic work. It was the contribution provided by these barefoot doctors that guaranteed rural people's basic medical treatment were stable in the situation that our economy was continuing to develop and people's living standard were falling behind. In the 1960s, China implemented the rural cooperative medical service system for the first time; it had been positively responded to by barefoot doctors. They had done a lot of work on health education, prevention and curing. Barefoot doctors were with highly enthused to serve the people all over the countryside, and they put prevention first and fought in the rural health, sanitation, epidemic prevention at the ground floor level of the three-level health care system for the prevention and cure of all types of infectious diseases, controlling of communicable diseases and endemics of many infectious diseases. They had successfully controlled epidemic encephalitis, measles and especially the spinal cord which had been sighted in the past decades.

Since the new-type rural cooperative medical service policies were implemented, under the direction of the government and the joint efforts of rural doctors, China currently has established a medical system network that nearly covers all of the countryside. It facilitates the peasants to see doctors, particularly for villagers who lived long away from hospital, and now they can go to the clinics for treatment, whenever. Meanwhile, the rural doctors responded to new-type rural cooperative medical service policy positively, and part of the village clinics' construction had been standardized. The medical equipment had been increased, the hardware had been improved, and the overall medical levels have been improved. Under relatively advanced equipment and sophisticated technologies', rural doctors provided professional medical examinations and treatment for the farmers and conducted follow-ups. The farmers' basic health care has been further protected. In short, during the implementation of the new-type rural cooperative medical service, under the joint efforts of the rural doctors, the burden of the villagers who take part in the new-type rural cooperative medical service policy has been relieved, the state of the treatment has been improved. They are playing an important role in promoting the economic and social development.

\subsection{The structure of the medical team}

The doctors in the new-type rural cooperative medical team consist of traditional rural coordinative medical and health treated barefoot doctors. They have been in the medical profession for many years and have built up the relevant medical work experience and knowledge.

\subsubsection{The age of a medical team}

Among the medical team, the sex ratio is fair balanced, but the overall age is trending to be much older. $44.4 \%$ of rural doctors' age is above 60 , while $33.3 \%$ is remaining between 50 and 59. The rest is distributed in 30 years to 49 years old, and the average age of the whole rural doctors has reached 56.8 years old.

\subsubsection{The education level of a medical team}

As more medical staff evolved from the barefoot doctors, most of them were lack of high education. According to our team in the Jiangxia district, Wuhan, the survey statistics showed that $50 \%$ of rural doctors were of technical education, and 10\% accepted high school education, while the remaining $40 \%$ had only junior education. Nationally, the ratio of the health workers who work in rural hospitals had a junior college degree or above the education was less than $23 \%$.

\subsubsection{Vocational education condition of the medical team}

The barefoot doctors should get the certificates to become the new-type rural cooperative medical service staff, among which, there were $33.3 \%$ who had received continuing education (assistant) doctor's certificate. According to the China Health Statistics Yearbook, about 60,000 of the general range medical practitioners practicing (assistant) doctors, only $3.5 \%$ of the total practicing (assistant) doctors. 


\subsubsection{Professional training of the medical team}

In the clinics which were researched, the rural doctors worked in the medical field average age was about 32 years old, and to obtain the relative titles on average 21 years. Meanwhile, these rural doctors would receive the relevant professional training, an average of 2.35 times per year.

\subsection{The development of the medical team}

Since 1950s, barefoot doctors have been the solid team which supported the primary caring, and they are also the pioneer and main force of the rural cooperative medical caring treatment. For now, barefoot doctors are still the main make up of the medicine care team. They are regarded as the foreign health care physicians, providing people with 24-hour, real-time personal medical services. According to our investigation, the rural doctors in Jiangxia district, Wuhan, take the same important role so whether there are enough and continuous human resources to fill the job vacancy decide the whole rural medicine care' development.

\section{The problems to the building of the new-type rural cooperative medical workforce}

\subsection{The serious ageing of the medical treatment team members}

\subsubsection{Hardship of one doctor one village (a doctor in a village)}

According to the data, the average age of the new-type rural cooperative medical treatment's fixed decimal staffs is 56.8 years old. Thus, the age of medical treatment team members are over sizing, and this condition may have an influence on the effectiveness of the new-type rural cooperative medical treatment' implementation. With the growth of the age, confined by the self-physical condition and the limitation of the medical knowledge, the ageing term will have influenced the sustainable practice of the medicine development. The existing villages almost have one clinic and one doctor. According to the research, among the existing rural doctors, 60 percent are barefoot doctors They are committed to the service of the substratum medicine .However, as they facing departure, the energy is limited. If the problem of successors were not assured, in that way, "one village one doctor" would not be effectively ensured in return.

\subsubsection{The hardship of adapting development}

The aging of the village doctor made themselves adapting development hard. It is mainly reflected in the following aspects:

2.1.2.1 The process of Modern Normalization is faster; the ability of accepting new things for rural doctors is lack of initiative and validity

To facilitate the concrete implementation of the new rural cooperative medical system at the grassroots level and the management of the cyber-networking medicine normalization, the state-designated new rural cooperative medical clinic must be equipped with computers and other related electronic equipment.These setouts are used to helping rural doctors effectively finish their work, such as the registration of the routine treatment work and treatment charges reporting. To some extent, whether rural doctors can effectively and skillful settle the Internet health care information using the electronic equipment decides the quality of the implement of the new rural cooperative medical system in the substratum.

According to our research, 40 percent of the rural doctors can skillfully handling the related information among the rural doctors, and the other 20 percent shows that they can handle the computers but not adept, and others show they even can't use computers, so they have to ask other people for help.

Due to the condition that the people in the village clinics are made up of rural doctors of older generation, they were born in the fifties and sixties last century, and have the common characters: convincing the traditional culture, having the commitment spirit, conservative ideas, weakness in accepting new things. With the growth of the age, the physiological problem that they are facing will be more protruding, including hearing and seeing weak and other factors, make them hard to handle the equipments. At the same time, most of the local government and health care institutions provide little related network technology training for rural doctors. Thus, they have to rely entirely on independent self-study, which resulted in the variations of their electronic devices operation level and cause the adequate quality of the work can not be guaranteed. The fact reflects that though related information equipment can be popularized in the basic level in theory, but the limitation of rural doctors' accepting the ability add constraints on it. Nowadays, informationlized time, the learning and operating ability can not keep pace with the new century development. Their experience and using of technology are so deficient that they are passively accepting new technology effectively. 


\subsubsection{More series of new-type diseases, rural doctors' medical ability reach bottleneck}

At the beginning of the $20^{\text {th }}$ century, SARS broke out. This disease caused high attention among both the party and government. The central and local government both mobilized people, "starting the battle against SARS immediately". As the main body of the basic health services at the grassroots level, rural doctors carried out the defense work in the substratum. At that time, people were lacking knowledge of new-type diseases and did not have effective prevention or cure measures, so did the rural doctors. In the view of the happen of the working bottleneck---weakness of dealing with unexpected situation, lacking knowledge of defending, personal defending measures not enough and the delay after getting the order from higher-ups, there are great challenges for rural doctors' working ability.

Nay, in recent years, due to the deterioration of the environment and climate, various zymosis infectious diseases and bird flu broke out. Also, nameless bacterium and virus are common occurrences, which are unpredictable and ferocious spread in a large range; whether rural doctors can manage the complex situation is a question.

2.1.2.3 New drug's coming out is going to be extremely intense, facing the difficulty of using and acquainting pesticide effect

With the areas expanding of medicine, new medicine's research and development have had became the areas that many company pay more attention to. In order to capture market and get the core competence, medical company's competence is going to be extremely intense. Some medicine has the same name but maybe from a different production area and the functions of the medicine are also different. The various medicines make the selling channel various, though the new-type rural cooperative medical treatment's fixed decimal medicine buying channel is single in theory, whether the buying channel is controllable, whether the rural doctors can appropriately use new various medicine to cure patients, are still problems. These problems will bring big challenges for rural doctors to distinguish the function of the medicine and medicine itself.

\subsection{The medical teams' quality need to be improved}

\subsubsection{Education}

Rural doctors' education level and their successors' education level have a direct influence on the quality of the new-type rural cooperative medical treatment. Among the doctors, about half are junior high school educational background; others are high school and technical secondary school. 56.7 percent don't have the qualification to have the exam to be a medical assistant, so we can distinguish, these rural doctors' educational level are relatively low. Nowadays, the doctors in medium size and small cities are graduated at technical secondary school at the minimum. By contrast, rural doctors' education falls far behind that of the doctors in the cities. Of course, we can't deny older generation barefoot doctors' medical level based on rich experience, but in the fast developing and information renewing times, just depending on the experience can not keep pace with the fast growth of the times.

\subsubsection{Professional education}

Compared with the continuous improvement of hardware conditions, Chinese new-type rural cooperative medical treatment staffs" "software" building is relatively weak; especially their professional education is lagging behind. On the basis of having the practice medicine qualification certificate, only one-third of the rural doctors passed the National Certified (Assistant), MD card, general medical practitioners (assistant) doctors are less and less. Among them, traditional Chinese general practice assistant surgeon is deficient. However, with the intensifying of the urbanization and the industrialization of our country, economic globalization, aging of population, the needs of the countryman to have high quality treatment are becoming higher and higher, having the higher demand for our medical teams, however, the deficient professional education is becoming the bottleneck of the new-type rural cooperative medical treatment. In fact, when farmers get sick, they are worried to come to the village clinic, but go to the province or city clinics, where they provide prevention, care, diagnosis, treatment, rehabilitation, health management integration of professional treatment and services. At this point, fostering high quality doctors have become very urgent task now.

\subsubsection{Professional training}

According to the 31 st in the chapter four of "Supervisory regulations for countryside medical practice," the provincial government, autonomous region and Municipalities organize and formulate the training plan of rural doctors, making sure the rural doctors at least receive one training every two years." During the interviewing of our group, we got the situation that the average training times organized by the local governments achieved about 2.35. And every training time is two to three days to ten days or half month. During the training, the government always arranges the students of the medical school to train the rural doctors, and the training content 
is about some medical professional skills. From the reflecting of some rural doctors, the training always skimping and the content is barren. And medical is a great event about the lives of people, so the training of the rural doctors should pay more attention.

\subsection{Needs improving for the welfare of the medical teams}

The welfare security problem of rural doctors has always been the focus of discussion. In public, the voice about improving the benefits of the rural doctors is becoming stronger. We compared rural doctors with teachers at the same level in rights and liabilities as well as welfare and remuneration. the average monthly income of the pointed New-type Rural Cooperative Medical treatment clinics’ staffs is only $¥ 1,230$, yet which is almost the single source of income. From its comparison with average operating capital per capita in the investigation--$¥ 23.300$, we can know the pressure of medical staffs. At the same time, Facilities subsidy of our government only benefit the clinic up to the standard, and the so-called subsidy is only limited to the bed, table, chairs, medicine and other simple equipments of disposable input. Other hardware facilities in clinics, such as housing, utilities and related working capital to buy drugs from the clinics are all responsible for personal commitment, which brought a big stress to rural doctors.

Since the implementation of New-type Rural Cooperative Medical treatment, according to the general reflection of medical staffs, the workload of medical doctors increases sharply: not only to complete the daily medical work but also the regular follow-up visit about the health conditions, from which the difficulty is countless, such as the communication barriers with the mentally ill patient. What's more, in New-type Rural Cooperative Medical treatment, the responsibility of medical accidents during common treatment is taken by medical personnel according to law? It was difficult for them to adapt because of the heavy work responsibilities and the risk of invisible pressure, besides, income returns and their responsibilities were very asymmetric, which leads people's satisfaction about the position of rural doctors get lower and lower.

In the aspect of attention and investment of government, rural medical fields do not get enough welfare security and proper attention compared to the cultural and educational profession. According to article 25 in Teaching Law in China, the average wage shall not be less than or should over than the average salary for national civil servants, and gradually raise, and establish a normal qualified for a higher wage system. According to Article 31, governments at each level shall adopt measures to improve the state and collective wage subsidies for the teachers working for primary and secondary school, Achieving equal pay for equal work of teachers in the equal level gradually. In contrast, the old rural doctors who had worked together with the teachers, they do not get regular pay and benefits of subsidies. However, they devote their whole life to medical profession with basically no holidays. After retiring, these medical workers also failed to get neither any social welfare safeguard, nor any system that feedbacks their sacrifice and preserves their rights and interests. All sorts of unfair treatment caused great distress to their elderly life. As a result, the rural doctors have to insist doing diagnoses from dawn to dusk.

As the diversification of modern society occupation, the employment has increased greatly. In the environment that the modern market mechanism is becoming gradually sound, the inheritance issue of rural medical clinic causes great concerns. Adjusting of both the welfare and remuneration and the proportion of rights and liabilities is extremely urgent.

\subsection{The development of the medical team is relatively difficult}

As mentioned before, the barefoot doctors are still right responsible to support rural medical system. Until 1977, the number of barefoot doctors had once reached 1.5 million, and up to 2008, the staff worked for village clinic had got 1.058 million. Meanwhile, a large number of medical university graduates step into the medical profession every year. However, the medical team still hasn't got enough "new blood", and sustains the original power; which needs to assimilate more persons of ability.

The investigation reflects the reality that most medical university graduates will not choose to work in poor or remote villages. This phenomenon is widespread, and the reason is also apparent. To some extent, the difference of the medical supplies and worker's treatment between city and country affects their employment choices. In cities, people tend to have good living conditions, allocation of public facilities, and more employment opportunities, which are attracting spacious graduates. The ultimate reason is the unbalanced situation among urban and rural development .But from a more direct level, the poor treatment of rural medical workers directly contribute to this issue.

The facilities in village clinic are at low level, and the clinic doesn't have perfect medical places .Besides, the low profit also cause little and little graduates to devote themselves to the rural medical career. In some clinics we find that some workers are the offspring of the barefoot doctors, these people in the village usually enjoy 
some reputation, but their medical ability is just so-so. Now, the older generation of the barefoot doctors have been almost in their fifties to sixties, the issue that there is whether a new batch of successor to replace them in five to ten years and whether any medical staffs to participate continually into this team in the future development process is related to the whole rural medical health system development.

\section{Countermeasures of developing the rural cooperative medical treatment teams}

\subsection{Improving the service capability}

Aimed to fulfilling the increased need for the professional knowledge of basic-level medical officers, it is necessary to strengthen the continuous education to the basic-level staffs. We suggest developing a long-term and effective training system, which is introduced as follows:

3.1.1 To dispatch the rural doctors in fixed days to counties or cities to have the professional courses and renew the knowledge, as well as the training about the technology, we can have a training every 3 months, a summary half a year, or an assessment annually.

3.1.2 To encourage the doctors in counties and cities to go to countries to communicate with the rural doctors about the professional technique in groups and cooperate with each others, which can carry out some activities in fixed days to exchange their experience, introduce the advanced ideas about the medical treatment, and at the same time, also bring inspiration to the medical mechanism in the county or city. In the cooperation, the rural doctors' ability is also improved and their horizon can be also opened.

3.1.3 There should be some related policies in related local governments and hospitals to ensure that these strategies work effectively. It can be contractual, which means that they can conduct a contract with the medical officers who are ready to go to countries. The contract should define when and where to go, as well as the responsibilities, and promise that during the period, the salary and welfare level should stay the same. What's more, it should not have a negative effect on the promotion in the future. We believe that there must be a lot of medical officers willing to take part in such a meaningful activity. Of course, it is indispensable to give an award and praise the medical officers. Over a long period of time, it can not only introduce talents to the village clinics to improve the medical treatment level, but also accelerate the development of the social graciousness about solidarity and mutual assisting, which can kill two birds with one stone.

Only through the scientific and systematic learning pattern can 'reproduce' large number of highly-qualified medical staffs and satisfy the villagers' increasing need which is willing to accept the advanced medical treatment. Meanwhile, only through the scientific and systematic learning pattern unsteadying of the traditional experience-passing on pattern can guarantee the sustainable development of the new-type rural cooperative medical treatment, and also ensure the continuous improving of the rural medical workforce's service capability.

\subsection{Introducing talents through various channels}

\subsubsection{To improve the training system of the rural doctors}

Aimed at the problem that the rural doctors mostly have under-average education, the government should attach importance to the rural doctors' training and perfect their training system. In practice, it can reinforce the building of the rural medical staffs through the method which to transplant the free training policies from the normal students to the rural doctors.

\subsubsection{The practicable measures are as below}

To set up some pointed medical universities to enroll the outstanding high-school graduates from the society, and encourage them to select the medical major of training for specific posts. Then after graduate, they come back where they came from to work for the village medical service.

3.2.1.2 The undergraduates who select the medical major of training for specific posts should sign the contract "Agreement on the free education for rural doctors", which must be performed strictly by the provincial-level administrative departments, the pointed subordinate medical universities and the graduates from the medical major of training for specific posts. The provincial-level administrative departments of the medical treatment are responsible for the employment of the undergraduates from the medical major of training for specific posts in its own administrative area, and ensure the policies are carried out so that the graduates can be a member of the medical staffs. In the meantime, they should establish the files about the good faith, and the records which are about breaking the contract public and record them into the files of the human affairs. In addition, they are also responsible for the management about the treaty giving-back and the penalty. 
3.2.1.3 Admitting the Apprenticeship Mode, cultivating younger generation for the new-type rural cooperative medical treatment

Aimed at the situation lack of rural doctors and aging of them, developing the Apprenticeship Mode is the most economical and the easiest way to cultivate the younger generation for the new-type rural cooperative medical treatment. The government promulgates the related policies, admitting and encouraging the old rural doctors to conduct the apprentices by themselves, and establishing the specialized assessment system to exam the apprentices' ability to work, and then to get the qualification certificate to take over the clinics. The Apprenticeship Mode stems from the ancient China and people are quite familiar with it. There is no need taking specialized propagating training and needs less capital. Taking these factors into consideration, the Apprenticeship Mode is an excellent solution to solving lack of rural doctors. What's more, with the promulgation of this mode, it can also partly settle the juveniles' unemployment.

\subsection{Improving the rural doctors' treatment and welfare}

In order to solve the problems caused by the unbalanced development between the cities and countries, it is essential to improve the working condition in the village clinics, the welfare and status of the medical staffs in order to stabilize the medical staffs.

\subsubsection{Reducing the working burden of the rural doctors}

The related local departments should enhance the assisting and management measures and disperses part of follow-up visits to the local family planning office and village office to reduce the working burden of the rural doctors, also, improve the quality of the follow-up visits. Furthermore, in order to stimulate the officers' passion and the working quality, there should be some extra award for the rural doctors who put on the duty of follow-up visits, for example, to give them extra allowance and the specific training, etc.

\subsubsection{Bring rural doctors into career preparation to make sure the supporting when they are getting older}

The government should provide rural doctors with much more favorable treatment and social status. It can bring rural medical officers into the nation's formal health workforce and into career preparation to reduce the differences between the teaching workforces with them gradually. For instance, the level of income should be equal to that of the local business units' workforce to guarantee their income for the daily life and also, the allowance for the holidays. What's more, it is a good idea to hold some community activities to enrich the medical workforces' spare time. In addition, it is indispensable to strengthen and improve the welfare when the rural medical workforces are retired; it is a wise way to bring the rural doctors' treatment into the government budget to make sure the supporting when they are getting older.

\subsection{Enhancing the building of rural medical workforce, and increasing the assessment and stimulation policies}

During the process of implementation of the new-type rural cooperative medical treatment, a large number of the medical teams are the core strength. Therefore, enhancing the building of rural medical workforce is of great urgency.

\subsubsection{Establishing the promotion system}

Establishing related promotion system is a meaningful method of stimulating the rural medical workforces' passion to take part in the training and autonomous learning. It can also encourage the people who had high-level education to accept the basic-level work and make a contribution to the rural medical treatment.

\subsubsection{Organizing the activity of changing quarters}

The government can arrange the medical staffs both in cities and in countries to join in the activities of relieving guard, which could enhance their communication about the medical technology and skills. It also does much good to their medical system because of the added new inspiration.

\subsubsection{Setting up the medical award and fund for scientific research}

The up-setter can be government or the social Commonweal or Charity Organization to set up the medical award and fund for scientific research for the innovation or breakthrough on the medical technology. In this way, it can encourage the rural medical staffs to innovate and attract medical staffs who work in cities to conduct researches in countries. And finally, it brings the rapid development of rural medical treatment.

\section{Final conclusion}

The new-type rural cooperative medical teams are the base of the new-type rural cooperative medical treatment system, as the dominant force which pays an immeasurable role. Only through enhancing the building and supporting of the medical staffs and improving their wholly capability, can sustain the medical mechanism at the 
present stage. Although we are quite far away from the expected situation, we believe that the difficulties in the process would be settled completely as such a benefiting policy is getting mature. And also, it can improve the service capability of the basic-level medical service mechanism gradually, and set up a dynamic base-level medical system and personnel mechanism for rural medical staff, which will finally grow up into a virtuous circle of the medical treatment system.

\section{References}

Experiments about New-type rural cooperative medical treatment assessment making group. (2006). The developing China's new-type rural cooperative medical treatment-report of New-type rural cooperative medical treatment pilot assessment program. Beijing: People's Press.

Health bureau information network in Jiangxia region. (2009). Wuhan. Jiangxia's New-type rural cooperative medical treatment clinics pilot program plan as a whole project.

History and status quo of rural medical, health economics, economics sub discipline, china economic network, and economic forum.

National development and reform commission, People's Republic of china. (2010). Suggestion regarding to enhancing the building of the rural medical workforce.

Sino-Japanese Cooperation Anhui province primary health care technology training project office. (2004). The new-type rural cooperative medical care and primary health care. Hefei University of Technology in Anhui.

$\mathrm{Xu}$, Songnan. New-type rural cooperative medical treatment pilot program of E Zhou city in Hubei province. People's New-type rural cooperative medical treatment daily online. 